\title{
Technique for accounting for the decrease in labor productivity due to workspace overcrowding
}

\author{
Vadim Undozerov ${ }^{1, *}$ \\ ${ }^{1}$ Moscow State University of Civil Engineering, 26 Yaroslavskoye Shosse, 129377, Moscow, Russia
}

\begin{abstract}
In civil engineering, delays are ubiquitous. They cause significant economic damage. One way to prevent deadline disruption is to lower the duration of tasks by increasing the number of workers on critical path activities. However, when the number of workers increases above a certain level, the duration stops decreasing due to the interference between workers that occurs in a limited work space. This situation is called "work space overcrowding". To determine the number of workers at which work space overcrowding occurs, as well as to improve activity scheduling accuracy, the author has developed a technique for accounting for labor productivity decline due to work space overcrowding. During the development of this technique, an analysis of existing approaches was carried out. Their shortcomings in practice are revealed. Then, the factors of productivity decline associated with overcrowding are identified. These factors are divided into two categories: "spatial" and "sociopsychological". Two models are developed based on a mathematical formalization of these categories. The criterion for choosing between the two models is the intensity of workers' movements. The technique was successfully verified using peer review by specialists in nuclear power plant construction. It was found that the technique accurately reflects the general nature of labor productivity dependence on the number of workers: constant levels of productivity up to a certain number, followed by decreasing levels of productivity, at accelerating rates, above that number. However, application of the technique provides only a rough approximation of this dependence in quantitative terms. This is compensated for by the technique's simplicity, requiring a limited number of inputs to produce a meaningful result. Finally, applying the technique to two specific cases, a recommended limit of the increase in the number of workers is determined for both. In the future, the technique can be applied to a wider range of construction activities.
\end{abstract}

\section{Introduction}

The actual construction duration is often significantly longer than planned [1-3]. It is accompanied by huge economical damage due to freezing investment, payback point delay, etc. [1] For nuclear power plants (NPPs) construction, the problem is of particular

\footnotetext{
* Corresponding author: und-vadim@yandex.ru
} 
importance [4-6]. Many modern NPP construction projects are characterized by significant delays (figure 1). Due to delays, the cost of these projects are often more than 2 times (4-5 billion dollars) bigger than planned [7,8]. The reasons of delays are delivery disruption [1], project participants' interaction problems [1], rework caused by design changes [9] or poor quality of task execution $[1,4]$, etc.

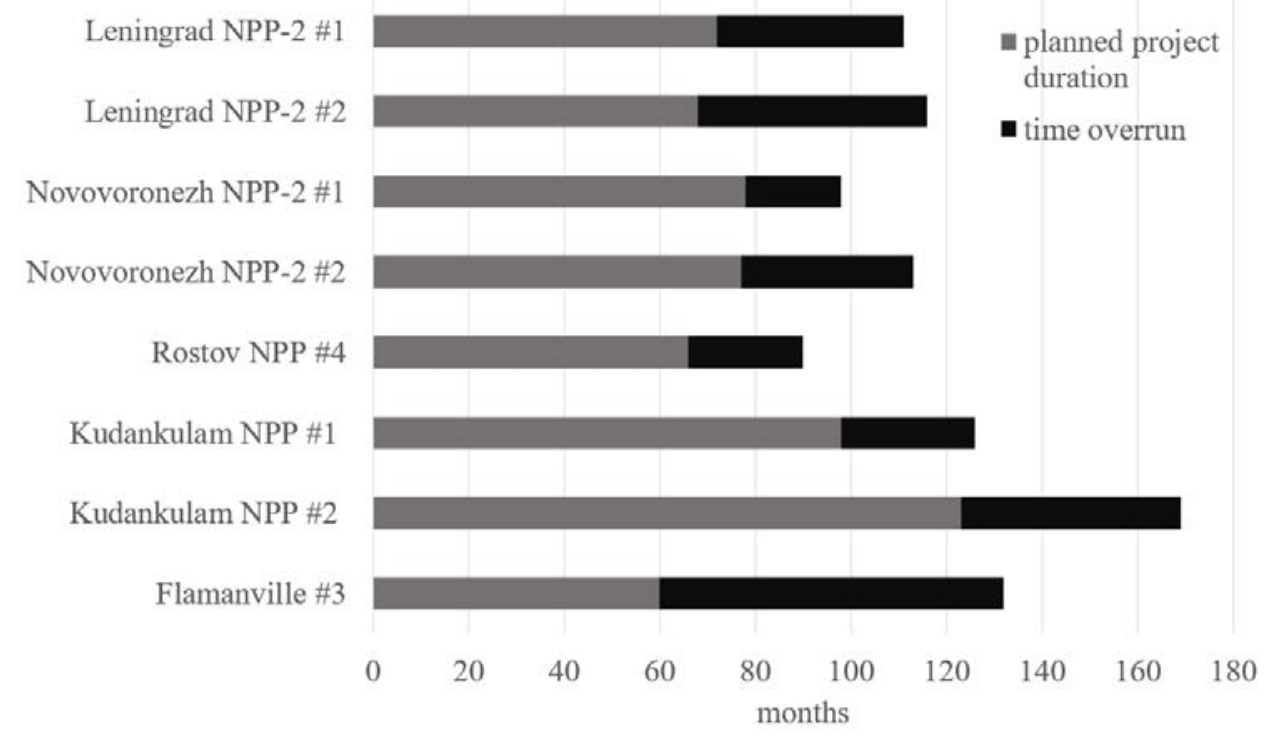

Fig. 1. Planned project duration and time overruns in some modern NPP construction projects. "\#" means a number of a power unit. The data was summarized by Achmetgaisin.

One common way to compensate for delays is to increase the number of workers on critical path activities. However, as work space is always limited, interference between workers occurs $[10,11]$. As a result, labor productivity decreases. Nevertheless, the total team output (TTO) initially increases due to the number of workers. It is so until some number of workers at which TTO reaches a maximum and begins to decrease. This number can be considered the limit (optimum), and the numbers above it can be called "work space overcrowding". Here and further, we mean optimality only in terms of reducing activity duration, when delay can lead to damage much greater than the losses associated with additional workforce involvement. Such factors as shortage of labor resources, degree of mechanization, etc. are not considered.

Accounting for the decrease in labor productivity due to overcrowding is also important to construction activity scheduling, since it can improve the duration assessment's accuracy. The lack of accuracy leads to the necessity of frequent rescheduling during the construction phase. This is a significant problem, especially for complex projects such as NPP construction.

Several techniques have been proposed to account for the phenomenon in question [12]. Their shortcomings in theoretical justification and/or narrow focus were revealed [12]. Since these drawbacks do not allow one to apply these techniques in practice, the author set and achieved the goal of developing a new, more advanced one. This technique is based on the research of such authors as M. Ringelmann, A.G. Ingham [13], B.V. Lukanin, I.V. Kim, G.L. Isaeva [12], U.-C. Klehe [12], J. Lee [15], A.A. Lapidus [16], I. L. Abramov [11] and others.

The remainder of this article is structured as follows. The section "Methods" presents the theoretical foundations of the technique, describes in detail the methodology and the 
course of the study. The section "Results" shows the deduced regularities, the most important mathematical formulas of the technique, as well as the results of its verification by peer review. In the "Discussion" section the assessment of the technique's validity and degree of accuracy is presented, as well as directions for future research are outlined. The "Conclusion" summarizes the main results of the study.

\section{Methods}

As a result of the previously proposed techniques' analysis [12], their common and different features were identified. The common is that the average labor productivity per worker (ALPW) is considered constant until a certain number of workers, and monotonously decreasing after that. The difference is in the functions describing this reduction. It was found that some of the proposed functions (models) are not theoretically or empirically substantiated enough. For example, the models proposed by Lukanin and Kim [12] suggest that ALPW decline slows down with increasing numbers of workers. It is contrary to practice, because it is evident, that the more people are already in the work space, the more detrimental is adding new ones. So, the decline in productivity should be not slowing down but accelerating. Then, the model proposed by Isaeva [12] is based on such assumptions as independence of the duration of activity's deployment and finishing from the whole activity duration, as well as labor productivity decline at the end of the task. These disputable assumptions were not proved. Other techniques are focused on narrow tasks (e.g., low-height construction [10]), without the possibility of expanding the application area [11]. Above drawbacks prevent the use of the existing techniques in practice. In order to overcome this issue, the objectives were set: 1) to develop more theoretically justified functions (models); and 2) to test them empirically.

To solve the first problem, the factors of labor productivity decline in the construction industry were analyzed by studying the literature and interviewing NPP construction specialists [16]. The factors associated with work space overcrowding were revealed, namely: 1) insufficient individual workplace's area for normal work; 2) reduced motivation; 3) reduced labor discipline; 4) reduced work quality; 5) sanitation deterioration. The factors are divided into two categories: simple "spatial factor" (downtime due to lack of space) and generalized "socio-psychological factor" (a set of the factors associated with reduced workers' motivation and personal responsibility). The nature of the interaction between the factors and the dynamics of their negative impact on labor productivity is analyzed. The factors are formalized in two models of productivity dependence on the number of workers - "hyperbolic" and "cubic".

The hyperbolic model is applicable for the activities with a large number of workers' movements (e.g., plates' reinforcement). The basic assumption of the model is that in this case the intensity of both factors is determined by the number of interactions between workers, rather than the number of workers per se. We mean the interactions related to communication (socio-psychological factor) and to the physical movements which cause individual workplaces' crossing, resulting in downtime (spatial factor). There is a reason to assume that, up to a certain number of workers, mainly socio-psychological factor acts, then both. Besides, the spatial factor increasingly "catalyzes" the action of another one with an increase in the number of workers. Therefore, the dynamics of labor productivity decline can be described, for example, using the hyperbolic growth function (general form $f(x)=\frac{C_{2}}{C_{1}-x}$, where $C_{1}, C_{2}$ - parameters, $x$ - variable), which is characterized by an increasing derivative with "aggravation". This "aggravation" occurs when all factors begin to act actively. As an additional justification for the "hyperbolic" hypothesis, an analogy 
from some researches in demography is used $[12,17]$. The hyperbolic growth function is used there to describe similar processes that depend on the number of relationships in system.

The cubic model is focused on the activities with a relatively small number of workers' movements, when they can be ignored (for example, columns' reinforcement). In this case it is feasible to assume the spatial factor's intensity to be proportional to the number of workers per se, while the socio-psychological factor's intensity - to the number of interactions (links) between workers. The maximum possible number of links is determined by the combinatorics formula: $n \cdot(n-1)$, where $n$ is the number of elements (here - workers).

Hyperbolic and cubic models allow one to determine the optimal number of workers (for given cases), which corresponds to the maximum TTO (in other words, the minimum activity duration). This is done by equating the TTO's derivative to zero. In the cubic model, the problem is reduced to solving a cubic equation. The Cardano's Method is used to solve such equations. In this method the equation $A \cdot x^{3}+B \cdot x^{2}+C \cdot x+D=0$ is reduced to the form:

$$
\begin{gathered}
y^{3}+p \cdot y^{2}+q=0, \\
y=x+\frac{B}{3 \cdot A} ; p=\frac{3 \cdot A \cdot C-B^{2}}{3 \cdot A^{2}} ; q=\frac{2 \cdot B^{3}-9 \cdot A \cdot B \cdot C+27 \cdot A^{2} \cdot D}{27 \cdot A^{3}} .
\end{gathered}
$$

The real solution of the equation is following:

$$
x_{0}=\sqrt[3]{-\frac{q}{2}+\sqrt{Q}}+\sqrt[3]{-\frac{q}{2}-\sqrt{Q}}-\frac{B}{3 \cdot A}, \text { where } \mathrm{Q}=\left(\frac{p}{3}\right)^{3}+\left(\frac{q}{2}\right)^{2}
$$

Peer review was used for the technique's verification. Observation on construction sites and production experiment had also been considered as alternatives. However, by observation one might obtain data about labor productivity only on a very narrow range of the number of workers. But it our case it is necessary to determine it on a wide range, from "normal" to almost maximum possible. Besides, using data from different sites would reduce the rigor of the empirical results. Production experiment would require significant financial, labor, and material resources, which was not expedient for initial verification. Since the initial verification by peer review turned out to be successful, conducting such experiments with the aim of more accurate verification may be the next step.

\section{Results}

Based on the hypotheses presented in the previous section, a technique for accounting for the decrease in labor productivity due to work space overcrowding was developed and tested. It is implemented as follows. If an activity is characterized by a large number of workers' movements, the hyperbolic model of labor productivity dependence on the number of workers is used to find optimal number of workers, as well as to calculate ALPW, TTO and activity duration. If the number of movements is insignificant and they can be ignored, the cubic model is applied to these purposes. The hyperbolic and the cubic models are described in detail below.

The hyperbolic model. According to the reasoning in the previous section, in the hyperbolic model the intensity of both factors (spatial and socio-psychological) has the same nature: it is determined by the number of interactions between workers. Thus, the factors are formalized integrally. 
The ALPW $(P)$ dependence on the number of workers $(n)$ is described by the formula (see "Methods"):

$$
P(n)=P_{0} \cdot \frac{C_{1}-n_{l}}{n_{\max }-n_{l}} \cdot(1-N(n))=P_{0} \cdot \frac{C_{1}-n_{l}}{n_{\max }-n_{l}} \cdot\left(1-\frac{C_{2}}{C_{1}-n}\right)
$$

where $P_{0}\left(\frac{\text { production units }}{\text { persons } \times \text { time unit }}\right)$ is the nominal ALPW (when there is no interference between workers); $n_{l}$ (pers.) - "productivity constancy limit": the maximum number of workers, at which the ALPW is constant and after which it begins to decrease; $n_{\max }$ (pers.) - the maximum number of workers at which it becomes almost impossible to work in a given space (ALPW becomes almost zero). $N(n)=\frac{C_{2}}{C_{1}-n}$ - the function reflecting a decrease in labor productivity due to the increase of the number of workers (figure 2). According to the arguments given earlier, it has the form of the hyperbolic growth function with the variable $n$ and the mathematical parameters $C_{1}$ and $C_{2}$. The parameters determine the nature of the decrease in labor productivity (the shape of the graph). The parameter $C_{1}$ represents the vertical asymptote of the graph (hyperbola). Its value depends on the work space area and the kind of work. The condition is imposed on it: $C_{1}>n_{\max }$. The higher this parameter's value, the flatter the graph is (the decrease in productivity is less "sharp"). The second parameter of the model $C_{2}$ is expressed as: $C_{2}=C_{1}-n_{\max }$, which follows from the boundary condition

$$
P\left(n_{\max }\right)=P_{0} \cdot \frac{C_{1}-n_{l}}{n_{\max }-n_{l}} \cdot\left(1-N\left(n_{\max }\right)\right)=P_{0} \cdot \frac{C_{1}-n_{l}}{n_{\max }-n_{l}} \cdot\left(1-\frac{C_{2}}{C_{1}-n_{\max }}\right)=0 .
$$

The multiplier $P_{0} \cdot \frac{C_{1}-n_{l}}{n_{\max }-n_{l}}$ is an expression of the horizontal asymptote of the graph of ALPW versus number of workers (figure 3 ). It is obtained from the boundary condition:

$$
P\left(n_{l}\right)=P_{0}=P_{a} \cdot\left(1-\frac{C_{2}}{C_{1}-n_{l}}\right) .
$$




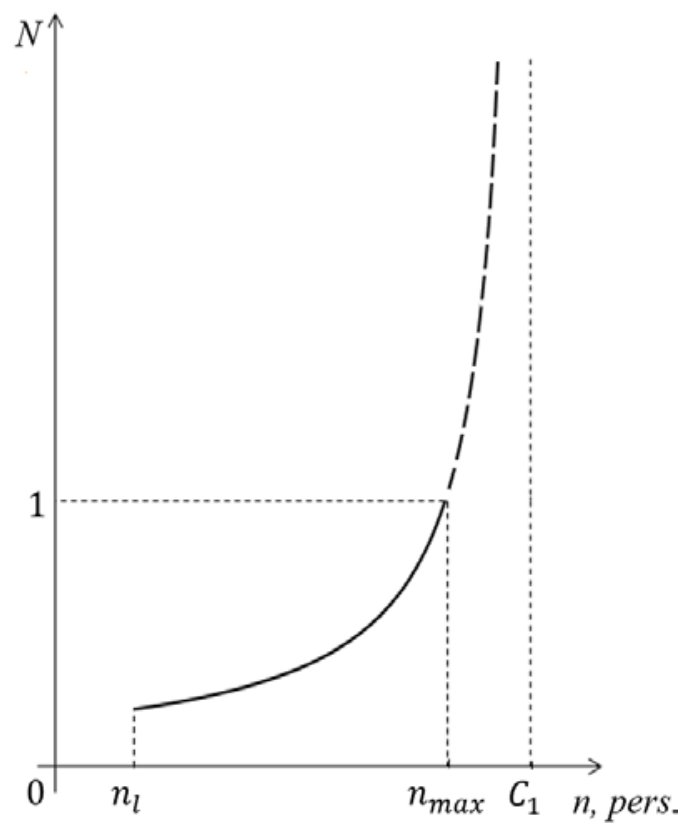

Fig. 2. The function that reflects the decrease in labor productivity due to the increase of the number of workers in a limited work space (solid line), in a hyperbolic model

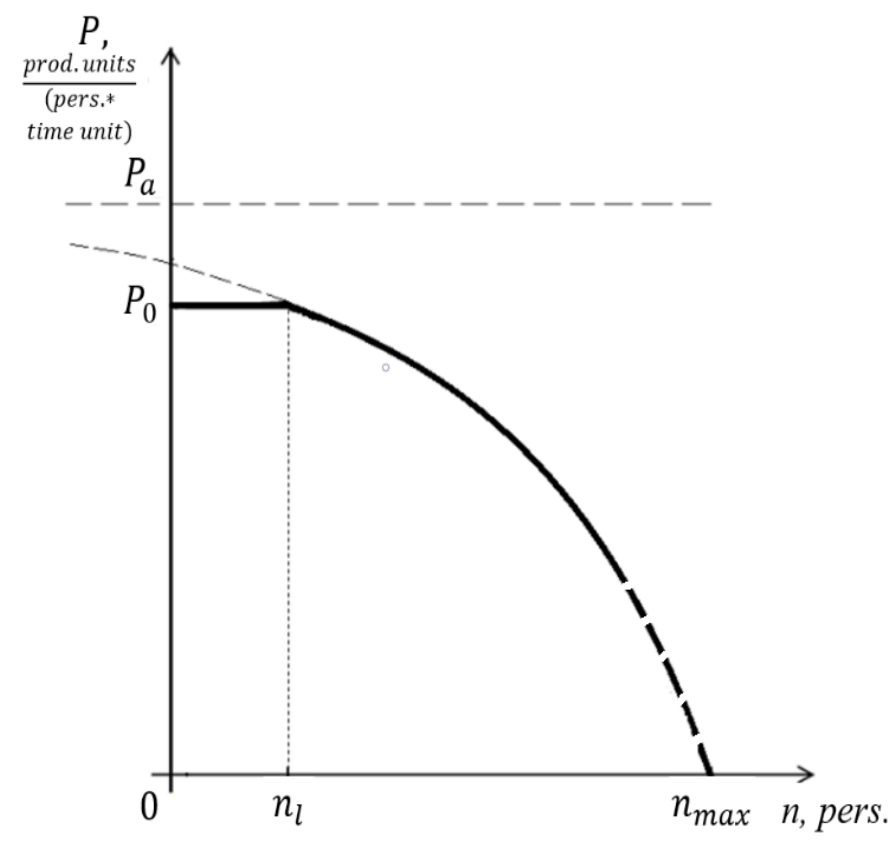

Fig. 3. The ALPW dependence on the number of workers (hyperbolic model).

Taking into account the above expression $C_{2}$ from $C_{1}$, the formula for the ALPW dependence on the number of workers (2) takes the form: 


$$
P(n)=P_{0} \cdot \frac{\left(C_{1}-n_{l}\right) \cdot\left(n_{\max }-n\right)}{\left(n_{\max }-n_{l}\right) \cdot\left(C_{1}-n\right)}
$$

The parameter $C_{1}$ (pers.) is determined by the known value of ALPW $P\left(n_{0}\right)$ at some number of workers $n_{0} \in\left(n_{l}, n_{\max }\right)$ by substituting these values into the formula (3):

$$
C_{1}=\frac{P\left(n_{0}\right) \cdot n_{0} \cdot\left(n_{\max }-n_{l}\right)-P_{0} \cdot n_{l} \cdot\left(n_{\max }-n_{0}\right)}{P\left(n_{0}\right) \cdot\left(n_{\max }-n_{l}\right)-P_{0} \cdot\left(n_{\max }-n_{0}\right)}
$$

TTO is determined by multiplying the ALPW by the number of workers. The idea of the TTO dependence on the number of workers is shown in figure 4.

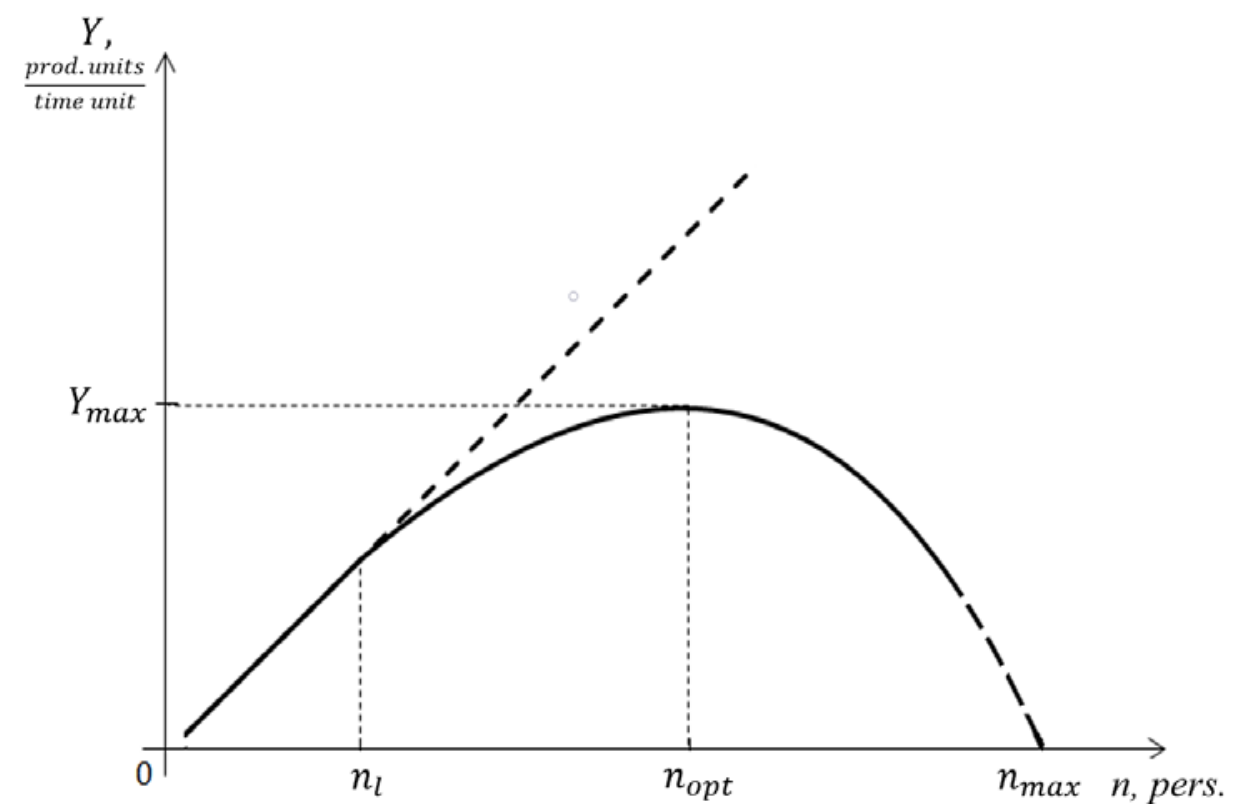

Fig. 4. The TTO dependence on the number of workers (hyperbolic model). $Y_{\max }$ is maximum TTO.

The optimal number of workers $n_{\text {opt }}$ (figure 4), which corresponds to the maximum TTO, is determined by equating the TTO's derivative to zero:

$$
n_{\text {opt }}=C_{1}-\sqrt{C_{1} \cdot\left(C_{1}-n_{\max }\right)}
$$

The cubic model. As mentioned earlier, in the cubic model, the characters of the sociopsychological and the spatial factor are assumed to be different. The intensity of the first is proportional to the number of connections between workers, while the intensity of the second is proportional to the number of workers per se. Therefore, the factors' formalization is performed separately, with subsequent integration.

The expression of the ALPW dependence on the number of workers is following:

$$
P(n)=P_{0} \cdot(1-N(n)), \quad n \in\left[n_{l}, n_{\text {max }}\right]
$$


where $N(n)$ is a function that reflects productivity decrease. It depends on the functions representing socio-psychological $\left(N_{1}(n)\right)$ and spatial $\left(N_{2}(n)\right)$ factor:

$$
N(n)=k_{1} \cdot N_{1}(n) \cdot N_{2}(n),
$$

where $k_{1}$ is a certain coefficient of proportionality. Functions $N_{1}(n), N_{2}(n)$ are multiplied because factors act simultaneously and affect each other. This corresponds to the approach used in the regulations when accounting for the coefficients reflecting various adverse conditions: hazardous work, high temperature, etc. The value $N_{1}(n)$ is assumed to be proportional to the maximum possible number of links, $N_{2}(n)$ - to the number of workers:

$$
N_{1}(n)=k_{2} \cdot\left(n-n_{l}\right) \cdot\left(n-n_{l}-1\right) ; \quad N_{2}(n)=k_{3} \cdot\left(n-n_{l}\right),
$$

where $k_{2}, k_{3}$ (pers. $^{-1}$ ) are coefficients that take into account weight of the factors. The shift to the right by an amount $n_{l}$ is because this value is the point when productivity reduction begins.

Then, the expression (6) takes the form:

$$
P(n)=P_{0} \cdot\left[1-k_{1} \cdot k_{2} \cdot k_{3} \cdot\left(n-n_{l}\right)^{2} \cdot\left(n-n_{l}-1\right)\right]
$$

According to the boundary condition $P\left(n_{\max }\right)=0$, the formula for the ALPW dependence on the number of workers takes the form:

$$
P(n)=P_{0} \cdot\left[1-\frac{\left(n-n_{l}\right)^{2} \cdot\left(n-n_{l}-1\right)}{\left(n_{\max }-n_{l}\right)^{2} \cdot\left(n_{\max }-n_{l}-1\right)}\right]
$$

Since expression (10) is a third-degree polynomial, the model is called "cubic". The general view of the dependencies in the cubic model is similar to the hyperbolic one, so one can obtain insights into these dependencies from the previously presented figures 3, 4 .

Finding the optimal number of workers was performed in the same way as when developing the hyperbolic model. Here the task was reduced to solving the cubic equation:

$$
A \cdot x^{3}+B \cdot x^{2}+C \cdot x+D=0
$$

with the parameters:

$$
A=4 ; B=-3 \cdot\left(3 \cdot n_{l}+1\right) ; C=2 \cdot n_{l} \cdot\left(3 \cdot n_{l}+2\right) ; D=-n_{\max }{ }^{3}+3 \cdot n_{\max }{ }^{2} \cdot n_{l}-3 \cdot n_{\max } \cdot n_{l}{ }^{2}+n_{\max }{ }^{2}-2 \cdot n_{\max } \cdot n_{l} \text {. }
$$

As a result of calculations, the formula of the optimal number of workers takes the form:

$$
\begin{aligned}
& n_{\text {opt }}=\sqrt[3]{B+\sqrt{Q}}+\sqrt{B-\sqrt{Q}}-\frac{3 \cdot n_{l}+1}{4}, \\
& B=\frac{-9 \cdot n_{l}^{3}-9 \cdot n_{l}^{2}+n_{l}-8 \cdot D-1}{64}
\end{aligned}
$$

$\mathrm{Q}$ - is a parameter in the Cardano's Method (see formula (1)). 
The technique's provisions were tested on two examples. These are tasks that lie on the critical path of NPP construction: the foundation and a floor plate's reinforcement in the reactor building. Both structures have round shape with diameter of about $50 \mathrm{~m}$ and thickness of 2.5-3 m. The tasks were chosen because the problem of labor productivity decline due to overcrowding is particularly important for critical path activities. Besides, these works have a significant manual labor component and are performed by large teams. The technique was used to calculate the duration and to find an optimal number of workers.

Peer review was used as the technique's verification method. The experts engaged into the peer review are NPP construction professionals who were selected based on several criteria, namely: 1) experience in the construction industry (minimum 5 years), 2) experience in the NPP construction (at least 1 year), 3) participation in execution or documentation development of the activities similar to that considered in the survey. The experts were asked to estimate the ALPW at different values of the number of workers, according to their experience and intuition. The TTO was determined by multiplying the ALPW by the number of workers. The results (see figure 5) were approximated by the formulas of the technique (3), (4), (10). The parameters in the formulas were obtained by iterating through all possible combinations of them and finding among them those at which they most accurately (in terms of the method of least squares) approximate the results of the peer review. The optimal values of the number of workers were determined by the formulas (5), (11). Calculations were performed using MatLab ${ }^{\circledR}$ software.
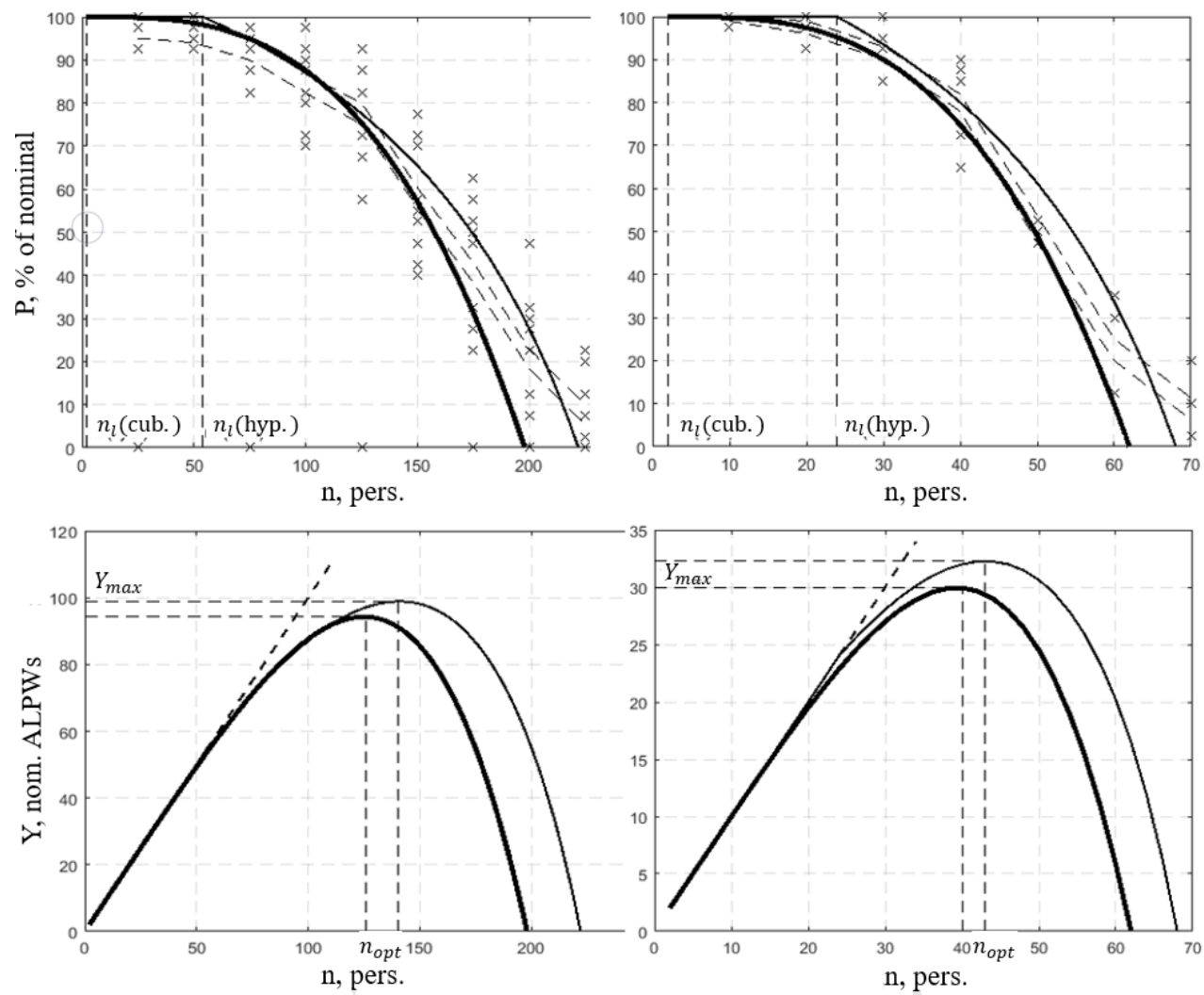

Fig. 5. At the left: the ALPW (top) and the TTO (bottom) dependence on the number of workers for the case of reinforcement the foundation plate of the NPP reactor building, according to: the peer review (x), the hyperbolic (thin solid line) and the cubic (thick solid line) model. By the dotted lines the corridor of average values of the peer review results are shown. At the right: the same as at the left, for the case of reinforcing a floor plate of the NPP reactor building. 
Based on the calculations made for the case of the foundation plate reinforcement, it is inferred that if it is necessary to accelerate this activity, the number of workers can be increased from the typical 50 up to $130-140$ persons. The result of this increase is reduction in the duration of NPP construction for up to 55 days (in the case of double-shift work schedule): from 120 to 65 days, with cost savings about \$ 8-50 million. The additional expenditure, mainly formed by required temporary buildings for workers, would be about $\$$ 300 thousand (according to Rosatom experts' estimates), which is negligible in comparison with the savings (3-4 orders of magnitude less). The average deviation of the theoretical values of labor productivity given by the technique from the corresponding empirical results of the peer review is $14 \%$ for the hyperbolic model and $27 \%$ for the cubic model.

\section{Discussion}

Higher accuracy of the hyperbolic model for the considered cases (piece reinforcement of plates) corresponds to the hypothesis that this model is more acceptable for the activities with a large number of workers' movements. At the same time, the cubic model also reflects the general nature of the dependence: the "accelerating" decrease in ALPW with an increase in the number of workers. However, both models have a rather large deviation from the empirical data. Therefore, the technique in its current form should be considered only as a rough model.

On the other hand, this roughness is compensated for by the technique's simplicity in terms of number and accessibility of the inputs. In the "cubic" model, there are only 3 input parameters - "nominal ALPW", " productivity constancy limit" and "maximum number of workers". The first parameter can be obtained from regulations or statistical data. The other two can be estimated by managers and other experts involved in a considered activity, according to their experience and intuition. It was found that the parameter "maximum number of workers" is difficult to assess directly. It is so because the condition when the number of workers is so high that ALPW drops to zero is impossible in practice. However, this parameter can be inferred from the expert's estimation of ALPW at some given number of workers ("a point: number of workers/ALPW") between productivity constancy limit and maximum number of workers. In this case experiment can be used as an alternative to expert estimates. In hyperbolic model, in addition to the above 3 inputs, another one is needed: a "point: number of workers/ALPW", to calculate the parameter $C_{1}$.

If there is a need for more accurate assessment of labor productivity, the technique can be developed, for example, by more detailed disclosure and study of factors categories. It's worth noting that alongside with ALPW one can use TTO and activity duration as the technique's metrics, since these variables are transformable into one another.

Although the technique can already be used in practice, there are still a great field for further research. First, more case studies are required to test the technique's validity. For now, the technique has been applied and successfully verified to only two activities. The next step could be the case study of the same activities in different context (e.g., other construction objects), then other activities might be considered. Besides, there are several important factors which weren't accounted for in the technique, such as simultaneous execution of two and more activities in the same work space, constraints related to cranes and other machines, managerial impact and some other. There is still the question about financial estimation of damage caused by delays, as well as about creation of systemic view on the problem of work space overcrowding. The latter means, in particular, that not only immediate, but also indirect consequences of overcrowding should be accounted for, e.g. phycological problems caused by accumulated stress and fatigue. 


\section{Conclusion}

The main result of the study is the technique for accounting for the decrease in labor productivity due to work space overcrowding. This technique allows one to find the optimal number of workers in the limited work space (for specific cases), at which activity duration is minimal. It is especially important when there is need to increase labor productivity after delays. The technique includes two models of the ALPW dependence on the number of workers - "hyperbolic" and "cubic". The criterion for choosing between the two models is the intensity of workers' movements. The technique was applied to two specific cases. As a result, a recommended limit of the increase in the number of workers is determined for both. It was found by peer review that the technique is valid in qualitative terms (at least for these cases), but rough in quantitative terms. This roughness is compensated for by the simplicity of the technique, namely requiring a small number and accessibility of the inputs to produce a meaningful result. The directions for future research were established: continuation of case studies and accounting for such factors as simultaneous tasks execution, machines' constraints, managerial impact, etc. The technique can already be used in practice as a rough model and can be applied to a wider range of construction activities after appropriate justification.

\section{References}

1. H. Doloi et al., Int. J. Pr. Man 30, 479-89 (2012)

2. A.H. Memon et al., J. Surv., Con. \& Prop. 2(1), 54-66 (2011)

3. M.A., Othuman Mydin et al., MATEC Web of Conf. 10, 06005 (2014)

4. T. Taylor et al., Proc. of the 23th International Conference of the System Dynamics Society (Boston, MA, 2005)

5. M.M. Hossen et al., Nucl. Eng. \& Techn. 47(3), 362-79 (2015)

6. S. Boarin, M.E. Ricotti, Proc. of the 16th International Conference on Emerging Nuclear Energy Systems (Spain, Madrid, 2013)

7. I.D. Sokolova, I.V. Blinova, Atom. Techn. Abr. 11, 11-24 (2011)

8. A.I. Solyanik, A.I. Kremenetskiy, Energ. Abr. 1, 12-19, (2019)

9. J. B. H. Yap, J. Surv., Con. \& Prop. 8(1), 13-33 (2017)

10. R.R. Remishev, Act. Probl. of Man. in Constr. Sect. 4, 126-136 (2010)

11. I.L. Abramov, Tech. \& Techn. 2, 110-13 (2006)

12. B. Pergamenshchik, V. Undozjorov, Econ. of Constr. 5, 25-34, (2018)

13. A. Simms, T. Nichols, J. Man. Pol. \& Pract. 15(1), 58-67 (2014)

14. U.-C. Klehe, N. Anderson, Int. J. Sel. \& Ass. 15(2), 250-62 (2007)

15. J. Lee et al., Aut. in Constr. 84, 121-132 (2017)

16. A. Lapidus, K. Tolstova, Ind. \& Civ. Eng. 5, 68-71 (2016)

17. B. Pergamenshchik, V. Undozjorov, E3S Web of Conferences 97, 06020 (2019) 